Rev Inv Vet Perú 2018; 29(1): 362-367

http://dx.doi.org/10.15381/rivep.v29i1.14192

\title{
Comunicación
}

\section{El Pitbull Peruano: una raza canina extinguida}

\author{
The Peruvian Pitbull: an extinct dog breed
}

\author{
E. Daniel Cossios ${ }^{1,2}$
}

\section{Resumen}

Durante la primera mitad del siglo XX, una raza de perro llamada Pitbull Peruano fue desarrollada en el departamento peruano de La Libertad. Este hecho fue reportado en un artículo en 1944 pero cayó en el olvido, al punto de no conocerse ninguna publicación posterior que nombre a esa raza. Con el fin de verificar si siguen existiendo perros con las características del Pitbull Peruano y de conseguir datos que permitan describirlo mejor, se realizó una búsqueda en la zona comprendida entre las antiguas haciendas Chiclín y Salamanca. No se encontraron ejemplares de la raza, pero ocho personas, de un total de 52 entrevistadas, proporcionaron nuevos datos sobre la historia y el aspecto de este perro. El Pitbull Peruano habría dejado de ser criado en 1964, siendo la única raza canina peruana de origen moderno de la que se tiene noticia y un elemento culturalmente valioso, particularmente para el departamento de La Libertad. Los datos aquí presentados permiten conocer mejor a este perro y podrían ser eventualmente utilizados para el establecimiento de su estándar y en la reconstrucción de la raza.

Palabras clave: estándar de raza; La Libertad; perro peruano; Pitbull Peruano; raza canina

\section{Abstract}

During the first half of the $20^{\text {th }}$ century, a dog breed called Peruvian Pitbull was developed at the Peruvian department of La Libertad. This was reported in an article in 1944 but fell into oblivion, to the point that there are not known later publications available which mention this breed. To verify if there still exist dogs with the Peruvian Pitbull traits and to get data which allow to better describe them, a search was carried on in the area

\footnotetext{
${ }^{1}$ Biosfera Consultores Ambientales, Lima, Perú

${ }^{2}$ E-mail:dcossios@yahoo.com
}

Recibido: 13 de junio de 2017

Aceptado para publicación: 9 de octubre de 2017 
between the former haciendas «Chiclín» and «Salamanca». No specimens of the breed were found but eight out of 52 respondents, provided new data on the history and aspect of this dog. The Peruvian Pitbull would have been ceased to be raised in 1964, being the only reported Peruvian canine breed of modern origin and a culturally valuable element, particularly for the department of La Libertad. The data reported here allows a better knowledge of this dog and could eventually be used for the standard establishing and reconstruction of the breed.

Key words: breed standard; dog breed; La Libertad; Peruvian dog; Peruvian Pitbull

\section{INTRODUCCIÓN}

En 1944 se publicó un artículo en el Journal of the American Veterinary Medical Association informando sobre una nueva raza de perro desarrollada en el Perú (McNellis, 1944), a la cual el autor llamó 'Peruvian pitbull'. El artículo menciona que la raza fue creada por la familia Larco, desde finales de los años 1910, en la hacienda Chiclín, ubicada en el departamento de La Libertad, con el fin de destinarla a la protección de los cerdos Poland China que criaban. El documento menciona también que se habrían criado unos 700 perros hasta estabilizar la raza y ofrece una corta descripción física de la misma. El artículo no describió las proporciones corporales de la raza, pero dio una escueta descripción de su carácter, indicando únicamente que «tenían la reputación de ser compañeros devotos de sus cuidadores y excepcionalmente eficientes guardianes de cerdos»». La raza nunca fue reconocida por la Federación Cinológica Internacional y cayó en el olvido, al punto de no conocerse ningún artículo u otro documento que la mencione, aparte del artículo de McNellis aquí nombrado.

Con el objetivo de verificar si continúan existiendo perros con las características descritas para el Pitbull Peruano y conseguir una mejor descripción de su carácter, historia y características físicas, se realizaron entrevistas a pobladores del área antiguamente ocupada por las haciendas de la familia Larco y se estudió la morfología de esta raza a partir de las fotos presentadas por McNellis (1944).

\section{Materiales y Métodos}

Con la intención de encontrar nuevas fotografías y otros documentos que pudieran mencionar al Pitbull Peruano, se contactó con el museo de Chiclín, ubicado en la localidad del mismo nombre, en el departamento La Libertad, Perú. Además, se hizo una visita a Chiclín y áreas aledañas para buscar perros con las características del Pitbull Peruano y entrevistar personas que pudieran conocer esa raza. La ubicación de Chiclín y otros puntos visitados se muestra en la Figura 1.

Las entrevistas, hechas de forma semiestructurada (Huntington 2000), incluyeron las siguientes preguntas: a) ¿Ha visto

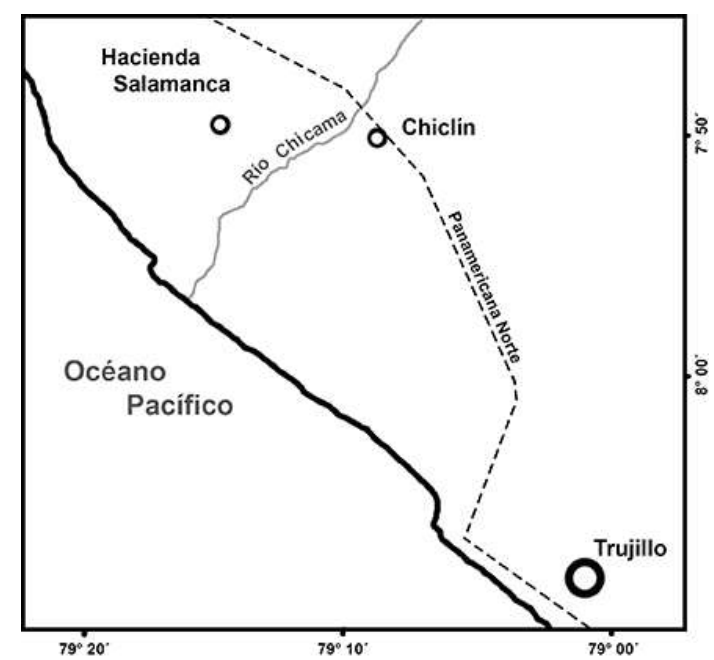

Figura 1. Mapa de ubicación del área visitada 


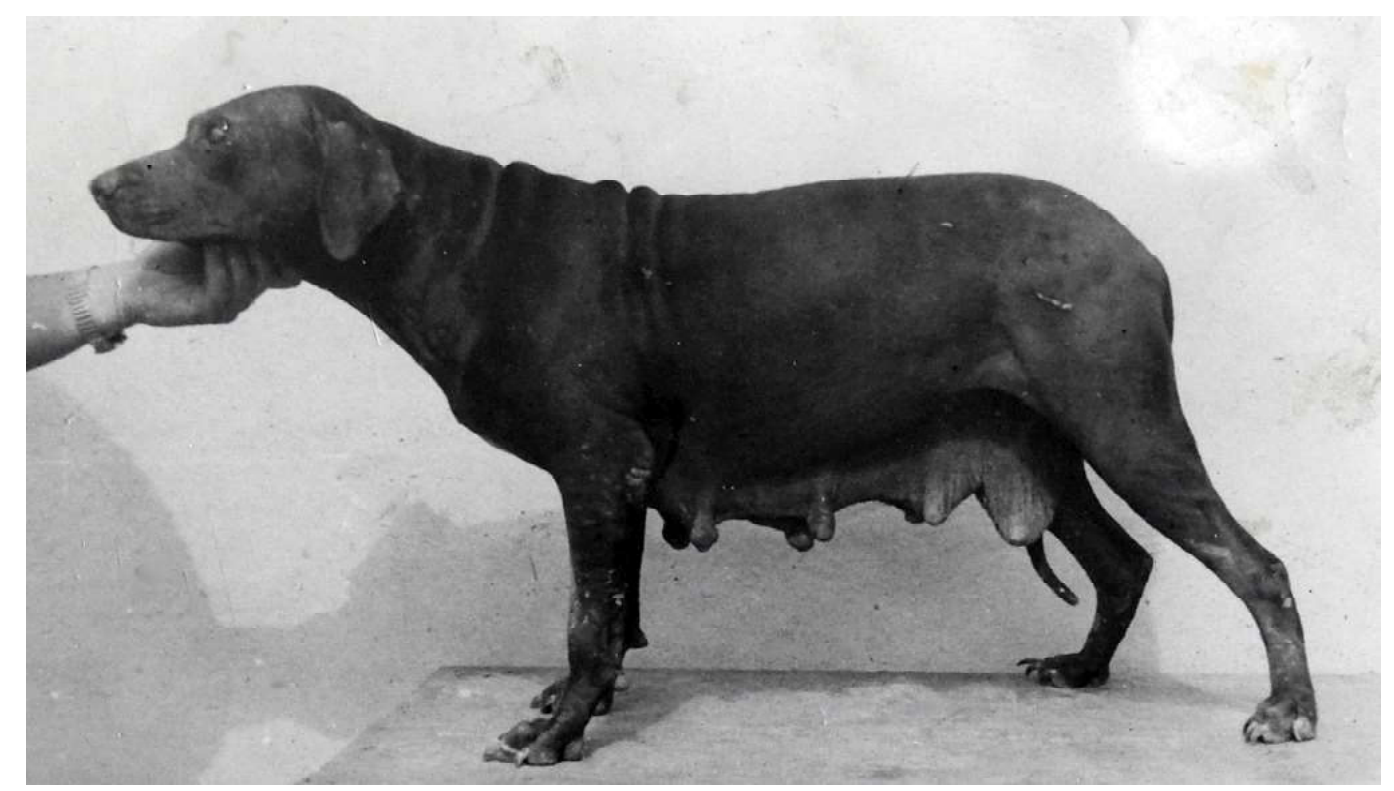

Figura 2. Hembra adulta de Pitbull Peruano. Fotografía encontrada en Chiclín, en posesión del señor Jorge Cox Denegri, y originalmente tomada y publicada por McNellis (1944)

perros como estos o conoce a alguien que los tenga?, b) ¿Dónde se criaba estos perros?, c) ¿Para qué se les utilizaba?, d) ¿Qué cuidados se les daba?, e) ¿Cuántos había?, d) ¿Tiene alguna foto de estos perros?, e) ¿Cómo eran físicamente? y f) ¿Cómo era su carácter? Aparte de las respuestas sobre estos temas, cualquier otro dato contado por los entrevistados fue anotado.

Para describir las proporciones corporales y la forma general de estos perros, se analizaron las fotos disponibles de ejemplares adultos de esa raza. De esta forma, se determinó las proporciones entre el largo y el alto del cuerpo, entre el largo de la cola y el cuerpo, entre el hocico y el cráneo y entre la cabeza y el cuello, y se describió la forma y posición de cola y orejas, la forma del pecho $\mathrm{y}$ los perfiles superior (dorso) y ventral del cuerpo.

\section{Resultados}

No se encontró ningún ejemplar de pero que cumpliera con las características físicas descritas por McNellis (1944) para el Pitbull Peruano. Se encontró una sola foto de estos perros (Figura 2), la cual es original y estaba en el museo de Chiclín. Esta foto era una de las presentadas por dicho autor. Debido a la ausencia actual de canes de esta raza y de nuevas fotos, las proporciones corporales y la forma general de esta raza fueron estudiadas exclusivamente a partir de las dos fotos de perros adultos del artículo de McNellis (1944).

En total, se entrevistó a 52 personas, 20 de ellas en la localidad de Hacienda Salamanca, 14 en Chiclín y 18 en áreas rurales situadas entre las dos localidades. Siete entrevistados recordaban haber visto perros 
de la raza, de estos, cuatro en Hacienda Salamanca y tres a dos km de Salamanca, cerca del poblado de Farías. Estas personas tenían más de 60 años, y habían vivido o trabajado en Hacienda Salamanca desde antes de 1960 .

En Chiclín, uno de los entrevistados fue el señor Jorge Cox Denegri, descendiente directo de la familia Larco y quien se encargó del museo de Chiclín hasta que este cerrara en 2012. Durante la entrevista, manifestó que los cerdos Poland China, ligados a los perros, no fueron criados en la Hacienda Chiclín, sino en la Hacienda Salamanca, lo que extendió la búsqueda a esa localidad. En Hacienda Salamanca, los cuatro entrevistados que dieron información útil vivieron toda su vida allí y recordaban muy bien a los perros. Uno de ellos estuvo encargado del cuidado de los cerdos Poland China. Los informantes de la zona rural entre Salamanca y Farías llegaron a la zona por trabajo o inmigrando con sus padres, entre 1950 y 1965 , y recordaban a los perros, aunque trabajaron en el cultivo de caña y no directamente con los canes.

De las entrevistas y revisión de algunos documentos, se consiguieron los siguientes datos históricos y descriptivos.

\section{Historia}

El Pitbull Peruano fue desarrollado como raza desde finales de la década de 1910 en la Hacienda Salamanca, situada en el departamento de La Libertad y propiedad de la familia Larco, con el objetivo de controlar a las ratas en las instalaciones dedicadas a la crianza de cerdos de raza Poland China (7/8 informantes), y no para cuidar a los cerdos como fue informado por McNellis (1944). En esa época, el administrador de la hacienda y posible promotor de la raza fue don Martín Lynch, a quien sucedió, en 1922, don Rafael Ventura (Larco Herrera, 1923). En los años 1950 llegó a haber más de 200 perros de esta raza en la hacienda (3/8 informantes), quie- nes tenían un cuidador y un cocinero a dedicación exclusiva (2/8 informantes). En 1964 se decidió abandonar la crianza de cerdos Poland China debido a razones de rentabilidad (2/8 informantes) y los Pitbull peruanos dejaron de ser seleccionados, mezclándose con perros mestizos ( $1 / 8$ informantes), lo que habría llevado a la extinción de la raza.

\section{Caracteristicas fisicas}

Color negro opaco (McNellis, 1944) o plomo oscuro ( $8 / 8$ informantes), con manchas blancas presentes en el extremo de las patas y/o en el pecho (5/8 informantes) en el $40 \%$ de los individuos (McNellis 1944). Piel gruesa y colgante, dando la apariencia de que se encuentra en exceso (5/8 informantes). Pelo corto y escaso, poco denso ( $3 / 8$ informantes).

- Orejas largas, anchas y caídas, frente ancha.

- Trufa oscura, labios colgantes. Cráneo mesocéfalo con depresión nasofrontal (conocida también como stop) pronunciada. Proporción cráneo: hocico de aproximadamente 2:1, con una longitud de hocico ligeramente menor a la mitad de la del cráneo.

- Cuello grueso y cilíndrico, aproximadamente del mismo largo que la cabeza.

- Cola larga, aproximadamente 2/3 del largo del cuerpo, de inserción baja, de buen grosor en el arranque y afilándose hacia la punta.

- Cuerpo mesomorfo, ligeramente más largo que alto, con una proporción aproximada de 1:0.83 entre el largo del cuerpo y la altura a la cruz.

- Altura a la cruz ligeramente mayor a la grupa. Perfil superior ligeramente cóncavo a la altura de la espalda y marcadamente convexo en la grupa.

- Pecho amplio y bajo, descendiendo un poco por debajo de los codos.

- El perfil inferior forma una línea recta a la altura del pecho hasta terminar las costillas, y sube ligeramente en la región ventral. 
- $\quad$ Altura a la cruz entre 45 y $51 \mathrm{~cm}$; peso entre 15.8 y $20.5 \mathrm{~kg}$ (McNellis 1944).

\section{Carácter}

Dócil, afectuoso con sus propietarios y cuidadores, curioso y despierto (6/8 informantes). Especialmente atraído por cazar roedores (3/8 informantes). Fácil de entrenar (2/8 informantes).

\section{Discusión}

Hasta el momento, el Perro sin Pelo del Perú es la única raza de perro de origen peruano que ha sido reconocida a nivel nacional, tanto por el Kennel Club Peruano como por la Federación Cinológica Internacional y otras instituciones. Diversos estudios arqueológicos sugieren que otras razas caninas pudieron haberse desarrollado antiguamente en el territorio peruano (Cornejo et al., 2012), incluyendo al llamado pastor Chiribaya, conocido únicamente por restos de animales momificados (Leonard et al., 2002).

Tanto el Perro sin Pelo del Perú como el pastor Chiribaya tienen un origen precolombino. El Pitbull Peruano es la única raza peruana de perro de origen moderno de la que se tiene noticia. Lamentablemente, su existencia pasó desapercibida fuera de su localidad de origen y, si no fuera por un artículo publicado a mediados del siglo XX (McNellis, 1944), hubiera caído en el olvido para siempre.

Debido al proceso de selección artificial que las moldea, las razas de animales domésticos son productos del ser humano $y$, como tales, deben ser consideradas parte del patrimonio cultural o histórico de sus países de origen (Ruane, 2000). Por esta razón, entre otras, las razas locales son actualmente objeto de importantes esfuerzos de conservación a nivel global (Ruane, 2000; Gandini y Villa, 2003). Aunque el Pitbull Peruano pare- ce actualmente extinto, su aspecto particular y su especialización como cazador de roedores lo muestran como un elemento culturalmente valioso, particularmente para el departamento peruano de La Libertad. Su creación, lograda a lo largo de varias décadas, es una muestra del desarrollo de la actividad agrícola y pecuaria en las haciendas peruanas durante la primera mitad del siglo XX y forma parte de su historia.

El cruzamiento es una de las principales causas de desaparición de razas de animales domésticos (Ruane, 2000) y puede llevarlas a la extinción en pocas generaciones (Mwai et al., 2015; Taberlet et al., 2008). La ausencia de ejemplares puros de Pitbull Peruano en la actualidad es entonces comprensible, dado que se le dejó de seleccionar hace 50 años, tiempo durante el cual las características de la raza se habrían diluido al cruzarse con perros de otras razas o mestizos.

Aun así, una raza puede ser recuperada o reconstruida a partir de ejemplares con características similares a las de la raza original. Ejemplos de reconstrucción de razas caninas son el Griffon Nivernais, considerado extinto a finales del siglo XVIII y recreado a partir de algunos ejemplares con características similares encontrados en su localidad de origen (FCI, 2004) y el Broholmer, casi extinto tras la segunda guerra mundial y recuperado a partir de unos pocos individuos con el fenotipo de la raza y ejemplares de otras razas (FCI, 2000; Pertoldi et al., 2013).

La ausencia de ejemplares vivos imposibilitó la redacción de un estándar completo para el Pitbull Peruano, faltando datos como el color de ojos, de trufa, ancho de cráneo y circunferencia de pecho, que no pudieron ser analizados en las fotos. A pesar de esto, los datos aquí proporcionados resultaron útiles para dar una descripción de la raza y podrían, eventualmente, servir como base para la reconstrucción de la misma y para el establecimiento de su estándar. 


\section{Agradecimientos}

El autor agradece a todas las personas que brindaron datos en campo y, en especial, al Sr. Jorge Cox Denegri, cuya información permitió dirigir la búsqueda en buena dirección.

\section{Literatura Citada}

1. Cornejo I, Pozzi-Escot D, Bernuy K, Angulo E, Tokuda LM. 2012. Hallazgos de Canis familiaris en el santuario de Pachacamac. Rev Haucaypata 5: 6-20.

2. [FCI] Fédération Cynologique Internationale. 2000. Broholmer, Standard FCI N. ${ }^{\circ} 315$. [Internet]. Available in: http://www.fci.be/en/nomenclature/ BROHOLMER-315.html

3. [FCI] Fédération Cynologique Internationale. 2004. Griffon Nivernais, Standard FCI N. 17. [Internet]. Available in: http:// www.fci.be/en/nomenclature/ GRIFFON-NIVERNAIS-17.html

4. Gandini G, Villa E. 2003. Analysis of the cultural value of local livestock breeds: a methodology. J Anim Breed Genet 120: 1-11. doi: 10.1046/j.14390388.2003.00365.x

5. Huntington HP. 2000. Using traditional ecological knowledge in science: methods and applications. Ecol Appl 10: 1270-1274. doi: 10.1890/1051-
0761(2000)010[1270:UTEKIS]2.0.CO;2

6. Larco Herrera R. 1923. 27 años de labor en Chiclín: reminiscencias y apuntes. En: Lima: Moral M. 247 p.

7. Leonard JA, Wayne RK, Wheeler J, Valadez $R$, Guillén S, Vilá C. 2002. Ancient DNA evidence for Old World origin of New World dogs. Science 298: 1613-1616. doi: 10.1126/science. 1076980

8. McNellis VC. 1944. The Peruvian bull terrier: new breed of dog. J Am Vet Med Assoc 104: 281-282.

9. Mwai O, Hanotte O, Kwon Y, Cho S. 2015. African indigenous cattle: unique genetic resources in a rapidly changing world. Asian Australas J Anim Sci 28: 911-921. doi: 10.5713/ajas.15.0002R

10. Pertoldi C, Kristensen TN, Loeschcke V, Berg P, Praebel A, Stronen AV, Proschowsky HF, Fredholm M. 2013. Characterization of the genetic profile of five Danish dog breeds. J Anim Sci 91: 5122-5127. doi: 10.2527/jas.2013-6617

11. Ruane J. 2000. A framework for prioritizing domestic animal breeds for conservation purposes at the national level: a Norwegian case study. Conserv Biol 14: 1385-1393.

12. Taberlet P, Valentini A, Rezaei HR, Naderi S, Pompanon F, Negrini R, Ajmone-Marsan P. 2008. Are cattle, sheep, and goats endangered species? Mol Ecol 17: 275-284. doi: 10.1111/j.1365294X.2007.03475.x 